\title{
Disclinations in Quasicrystals
}

\author{
J. Bohsung and H.-R. Trebin \\ Institut für Theoretische und Angewandte Physik der Universität, D-7000 Stuttgart 80, West Germany
}

(Received 10 October 1986)

\begin{abstract}
The most significant feature in the transition from the quasicrystalline to the amorphous state is the loss of long-range bond-orientational order. Disclinations are candidates for elementary excitations which destroy angular correlations. Generalizing the topological defect classification, we investigate point singularities in two-dimensional pentagonal quasicrystals and construct disclinations, dislocations, and disclination dipoles.
\end{abstract}

PACS numbers: $61.55 . \mathrm{Hg}, 61.50 . \mathrm{Em}, 61.70 . \mathrm{Ga}$

The Al-Mn alloy "shechtmanite," which exhibits a diffraction pattern of icosahedral symmetry, ${ }^{1}$ is commonly interpreted as a quasicrystalline state of matter. ${ }^{2}$ Soon after the discovery of this exceptional phase, Urban, Moser, and Kronmüller ${ }^{3}$ studied transitions from the quasicrystalline to the amorphous state of $\mathrm{Al}_{86} \mathrm{Mn}_{14}$ by electron irradiation. During the process a series of electron diffraction patterns was taken. These show several of the decagonal stars of sharp diffraction spots gradually developing into rings of the same radial distance. The results indicate that the local neighborhoods of the atoms are preserved while the angular correlations of different neighborhoods are perturbed.

The features remind us of the melting process of the two-dimensional hexatic phase proposed by Nelson and Halperin. ${ }^{4}$ The melting is initiated by the unbinding of disclination-antidisclination pairs. It is quite possible that disclinations are the relevant elementary excitations destroying the angular correlations in quasicrystals and thus driving the transition to the amorphous state. But what is a disclination in a quasicrystal?

To approach an answer to this question, we restrict ourselves to two-dimensional pentagonal quasiperiodic patterns and study their topologically stable point singularities-including disclinations. These patternsfrequently called Penrose ${ }^{5}$ patterns- have been analyzed in detail by de Bruijn ${ }^{6}$ and-in three-dimensional extension- by Mackay, ${ }^{7}$ Duneau and Katz, ${ }^{8}$ and Kramer and Neri. ${ }^{9}$

Topological defects are closely related to the symmetry of the perfect system. They are characterized by closed loops traversing the so-called order-parameter space $V .{ }^{10}$ For periodic crystals this space is found in the following way: At a point $P$ of the defected crystal a copy of the perfect medium is placed such that the two structures coincide locally. The order parameter at $P$ is constituted by the set of rigid-body operations, which move a uniform reference system into the position of the copy. This set is the coset $g H$, where $H$ denotes the space group of the perfect crystal and $g$ is an element of the unbroken symmetry group $G$. The group $G$ leaves the free energy of the system invariant. The order- parameter space itself is the coset space $G / H$.

Quasicrystals do not possess a space group, because they are not periodic, and we cannot specify the orderparameter space immediately. We have to investigate the symmetry properties with care, starting from the construction principles of a perfect Penrose pattern.

One method to obtain a Penrose pattern is the projection method, ${ }^{8,9}$ which we apply here in a slightly modified form. ${ }^{11}$ It is based on a hypercubic lattice $L_{\gamma}$ in the five-dimensional Euclidean space $E^{5}$. The lattice is shifted from the origin of $E^{5}$ by a vector $\gamma \in R^{5}: L_{\boldsymbol{\gamma}}=\boldsymbol{\gamma}+Z^{5}$. In $E^{5}$ a two-dimensional subspace $P_{T}$, the tiling plane, is embedded in such a way that it remains invariant under $C_{5}$, the group of cyclic permutations of the canonical basis vectors of $E^{5}$. Aside from $P_{T}$ the group $C_{5}$ leaves the diagonal $\Delta=(1,1,1,1,1)$ and and another two-dimensional subspace $P_{\perp}$ of $E^{5}$ invariant and divides $E^{5}$ into three subspaces: $E^{5}=P_{T}+P_{\perp}+\Delta$. By moving the unit cube $W^{5}$ along $P_{T}$ one cuts out a strip $S=P_{T}+W^{5}$. The projection of the union of all two-faces entirely contained in $S$ yields a tiling of $P_{T}$ of fivefold long-range bond-orientational symmetry.

With respect to a lattice point, the symmetry group $H_{L}$ of $L_{\gamma}$ is the semidirect product of the hyperoctahedral group $\Omega(5)$ and the translational group $Z^{5}$. If we consider all operations with respect to the origin of $E^{5}$, we have to conjugate $H_{L}$ with a translation $\{1, \gamma\}$ by the shift vector $\boldsymbol{\gamma}$. $H_{L}^{\text {conj }}=\{1, \gamma\}\left\{\Omega(5) \wedge Z^{5}\right\}\{1, \gamma\}^{-1} ; \wedge$ denotes the semidirect product. Our perfect system consists of three parts: $l_{\gamma}, S$, and $P_{T}$. By definition all symmetry operations act only on $L_{\gamma}$ (active viewpoint); $S$ and $P_{T}$ are fixed in $E^{5}$. Now we can state that $H_{L}^{\text {conj }}$ is "a symmetry group of the Penrose pattern," in the sense that a tiling projected from $L_{\gamma}$ is identical to one projected from $H_{L}^{\text {conj }} L_{\boldsymbol{\gamma}}=L_{\boldsymbol{\gamma}}$.

As in the case of periodic crystals we identify the order-parameter space with a coset space $V=G / H$. $G$ is the group which produces set of patterns of the same free energy. Such sets are the local ismorphism (LI) classes. ${ }^{12}$ The tiling projected from a lattice $g L_{\gamma}, g \in G$, is in the same LI class as the tiling projected from $L_{\boldsymbol{\gamma}}$, if the following conditions are fulfilled. 
(a) The vectors $\mathbf{t}$ in the translations $\{1, \mathbf{t}\} \in G$ are elements of the orthogonal complement of $\Delta$ in $E^{5}$, denoted by $R^{4}$ because the shift vectors $\gamma+\mathrm{t}$ of the lattices $\{1, \mathbf{t}\} L_{\gamma}=L_{\gamma+\mathrm{t}}$ must have the same projection onto $\Delta$ as $\gamma$.

(b) Certainly, pure rotations in $G$ must be compatible with the translations in $R^{4}$. So the rotational part $G_{0}$ of $G$ is a subgroup of the direct product $\mathrm{SO}(4)_{R^{4}} \otimes 1_{\Delta}$. Furthermore, the slope of $P_{T}$ with respect to the lattice must not change. Otherwise even the shape of the tiles is altered. It follows that $G_{0}$ equals $\mathrm{SO}(2)_{T} \otimes \mathrm{SO}(2)_{\perp}$ $\otimes 1_{\Delta} . G$ itself is the semidirect product $G_{0} \wedge R^{4}$.

The group $H$ has to be a subgroup of $G$, and so it cannot be the full symmetry group $H_{L}^{\text {conj }}$, but only the subgroup

$$
H=G \cap H_{L}^{\text {conj }}=\{1, \gamma\}\left\{C_{5} \wedge T^{*}\right\}\{1, \gamma\}^{-1} .
$$

$T^{*} \subset R^{4}$ is the group of those discrete translations of $Z^{5}$ which have no component along $\Delta$. An element $\mathbf{t}^{*}$ has the form $\mathbf{t}^{*}=\sum_{i=1}^{4} n_{i} \mathbf{a}_{i} ; n_{i} \in Z ; \mathbf{a}_{i}=e_{0}-\mathbf{e}_{i}$.

As in the case of normal crystals, every point in $V$ (i.e., every coset of $H$ in $G$ ) corresponds to a perfect system, which arises from a reference system by the action of any element of the coset. But in contrast to periodic crystals, two perfect quasiperiodic patterns which belong to different points in $V$ may not be matched by a rigid motion. They are only locally isomorphic and not globally. This is a consequence of the fact that in general the action of an element $g \in G$ on $L_{\gamma}$ changes the union of two-faces in $S$ and also the tiling itself.

More general defects can be constructed by choosing as order-parameter space $V=E(5) / H_{L}^{\text {conj }}$.

Point singularities in two-dimensional patterns are topologically classified by the fundamental group of the order-parameter space, $\pi_{1}(V) .{ }^{10}$ Because $H$ is discrete, $\pi_{1}(V)$ is isomorphic to the lift $\bar{H}$ of $H$ into the universal covering group $\bar{G}$ of $G: \pi_{1}(V)=\bar{H}=\{1, \gamma\}\left\{Z \wedge Z^{4}\right\}$ $\times\{1, \gamma\}^{-1}$. An element $r \in Z$ labels a rotation by $r \times 72^{\circ}$. An element $\left(n_{1}, n_{2}, n_{3}, n_{4}\right) \in Z^{4}$ marks a translation by the vector $\mathrm{t}^{*}=\sum_{i}^{4}={ }_{1} n_{i} \mathbf{a}_{i}$.

1. Dislocations. - If we consider only translational displacements in a pattern, we have to deal with a reduced order-parameter space $V=R^{4} / T^{*}$, which already has been investigated by Kléman, Gefen, and Pavlovitch. ${ }^{13}$

The defects are labeled by $\pi_{1}(V)=Z^{4}$. Each element $\left(n_{1}, n_{2}, n_{3}, n_{4}\right) \in Z^{4}$ corresponds to a Burgers vector $\mathbf{b}=\mathbf{t}^{*}$.

2. Disclinations. - A pure disclination corresponds to the conjugation class $\{1, \gamma\}\left\{r, T_{\text {sub }}^{*}\right\}\{1, \gamma\}^{-1}$ of an element $\{1, \gamma\}\{r, 0\}\{1, \gamma\}^{-1}$. $T_{\text {sub }}^{*}$ denotes a sublattice of $T^{*}$. It is spanned by the vectors $(1-r) a_{i}, i=1,2,3,4$ (details are described in a forthcoming publication; see also Ref. 10).

As an example we consider a $72^{\circ}$ disclination at the origin of $E^{5}$. The corresponding element of $\pi_{1}(V)$ is

$$
\{1, \boldsymbol{\gamma}\}\{r, 0\}\{1, \boldsymbol{\gamma}\}^{-1} ; \quad r=1 \text {. }
$$

Before discussing this disclination, we recall a disclination in a square lattice. It is classified by the element $\{r, 0\}$, where $r$ denotes a rotation about $r \times 90^{\circ}$. As an example, take $r=1$. Again we use the Volterra process, which now consists of the following two steps: (1) Cut out the third quadrant of the square lattice and identify the lip at $0^{\circ}$ and the lip at $270^{\circ}$. (2) Glue the two lips together by rotation of the lip at $270^{\circ}$, thus distorting the medium.

In a Penrose pattern, it is generally not possible to construct a disclination by cutting out or, in the case of $r<0$, adding a sector of the tiling, because there is no global fivefold symmetry (an exception is the highly singular case $\gamma=0$ ). How to proceed becomes clear if we rewrite Eq. (1) in the following way:

$$
\{1, \boldsymbol{\gamma}\}\{r, 0\}\{1, \gamma\}^{-1}=\{r, 0\}\left\{1, r^{-1} \gamma-\gamma\right\} \text {. }
$$

After one encircles the core of the defect, the lattice $L_{\gamma}$ has changed to a lattice $L_{\gamma+b}$. We have to choose a spatially varying shift vector $\gamma(x)$. In the simplest case $\gamma$ is only a variable in $\theta$, the polar angle in $P_{T}: \gamma(\theta)$ $=\gamma(0)+(\theta / 2 \pi) \mathbf{b}$ (see, for example, Lubensky, Socolar, and Steinhardt ${ }^{14}$ ). To construct a dislocated tiling, we have recourse to the Volterra process. For periodic crystals, it consists of two steps. (1) Remove a slice of matter corresponding to the Burgers vector and identify the lips. (2) Glue the lips together by distorting the lattice. If $\mathbf{b}$ is a lattice vector, they match.

It is straightforward to generalize this rule to the case of Penrose patterns. First we change from the former active viewpoint (unshifted strip $S$, shifted lattice $L_{\gamma}$ ) to an equivalent passive viewpoint: lattice fixed, strip translated by $-\boldsymbol{\gamma}$. Then we manipulate both the lattice $L$ and the strip $S_{-\gamma}$. In $L$, step (1) of the Volterra pro-

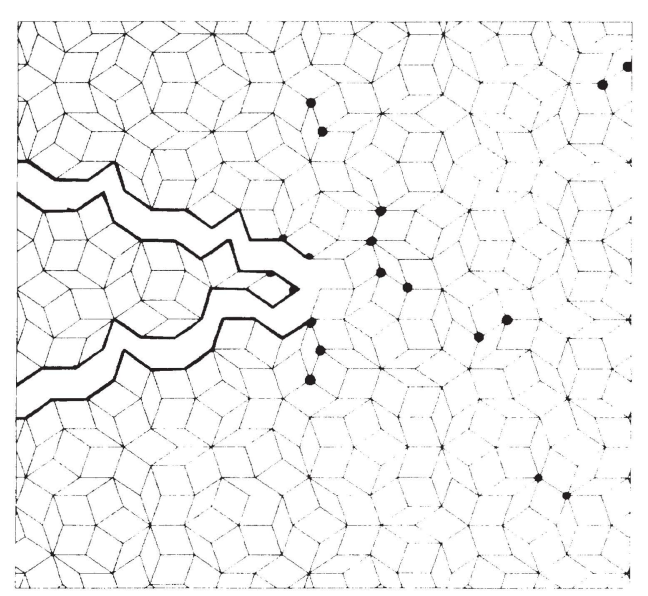

FIG. 1. Step (1) of the Volterra process for a dislocation of Burgers vector $\mathbf{b}=(0,1,0,0,-1)$. Vertices outside the LI class of the perfect tiling are marked by dots. Step (2) (lips glued together) is depicted in Ref. 14. 

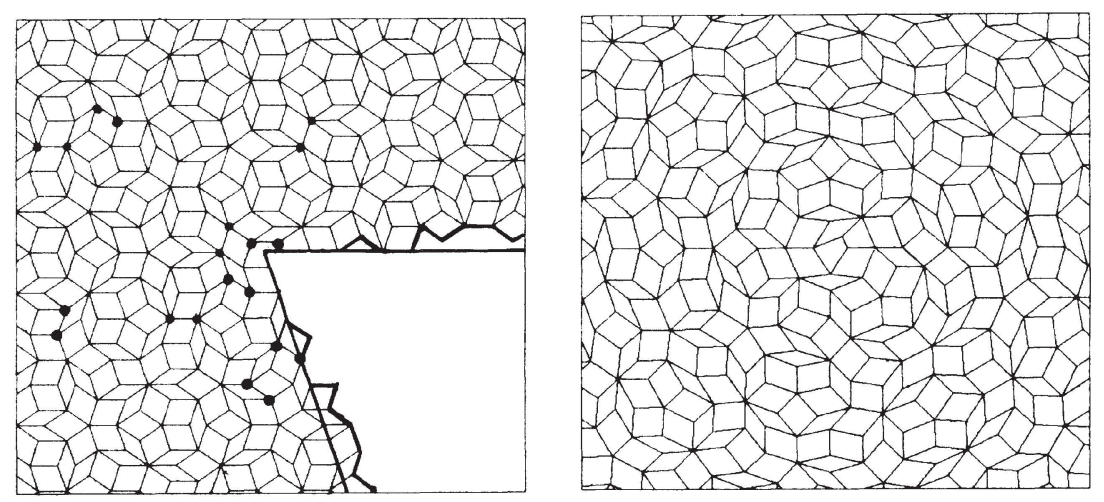

FIG. 2. $72^{\circ}$ disclination in a Penrose pattern. (a) Step (1) of the Volterra process. Vertices outside the LI class are marked. (b) The same disclination after closure of the sector by angular distortion.

cess is performed. Simultaneously, the strip $S_{-\gamma}$ is bent such that it interpolates between the plane strips $S_{-\gamma}(0)$ and $S_{-\gamma(0)-b}$ according to the spatial dependence of $\boldsymbol{\gamma}$. Figure 1 represents the first step of the generalized Volterra process. Removal of matter in $L$ alone would not be sufficient to make the lips match. An additional rearrangement of the tiles is necessary, which naturally occurs if we bend the strip.

Before the identification of the two lips (characterized by $\{r, 0\}$ ), we have to change $\boldsymbol{\gamma}$ into $\left\{1, r^{-1} \boldsymbol{\gamma}-\boldsymbol{\gamma}\right\} \boldsymbol{\gamma}$ $=r^{-1} \gamma$ between $\theta=0^{\circ}$ and $\theta=(5-r) \times 72^{\circ}$. A simple choice of $\gamma(\theta)$ for a $72^{\circ}$ disclination is the following:

$$
\begin{aligned}
\gamma_{i}(\theta)=\left(\theta / \frac{8}{5} \pi\right)\left[\gamma_{i+1}(0)-\gamma_{i}(0)\right]+\gamma_{i}(0) & \\
& i=0, \ldots, 4 .
\end{aligned}
$$

As in the case of the dislocation, we carry out step (1) of a generalized Volterra process by changing from the active to the passive viewpoint: From the unshifted lattice we remove a sector, and we use a curved strip $S_{-\gamma(\theta)}$. The resulting pattern [Fig. 2(a)] displays the rearrangement of tiles without distortions. In Fig. 2(b) the sector has been closed according to step (2).

3. Disclination-antidisclination pair. - The topological classification proves $^{10}$ that a disclinationantidisclination pair, separated by the projection of a lattice vector $\mathbf{t}$ on $P_{T}$, corresponds to a dislocation of Burgers vector $\mathbf{b}=(r-1) \mathbf{t} \in T^{*}$. The dislocation with $\mathbf{b}=(0,0,3,-3,0)$ shown in Fig. 3 , is equivalent to a $72^{\circ}$-disclination dipole. The cores are separated by $\mathrm{t}=(0,0,-3,0,0)$.

Topological defects in quasiperiodic patterns are accompanied by local neighborhoods of tiles (in the figures marked by dots), which do not belong to the LI class of the perfect pattern. These exceptional neighborhoods are denoted phasonlike defects or mistakes. ${ }^{15}$ Phasons are new elementary excitations, which in incommensurate structures appear in addition to phonons.

Mistakes are a consequence of the special order- parameter space: A loop in $V$ corresponds to many different patterns. In the defected state several perfect patterns are combined with the phasonlike defects serving as "adapter tiles."

Line singularities in icosahedral quasicrystals. When use the method of projection from a sixdimensional hypercubic lattice onto a three-dimensional tiling plane, all patterns belong to the same LI class. ${ }^{12}$ Therefore for the translational part of the unbroken symmetry group $G$ all translations in six-dimensional space are permitted. The rotational part of $G$ must leave the tiling space invariant; hence $G=\left\{\mathrm{SO}(3)_{T} \otimes \mathrm{SO}(3)_{\perp}\right\}$ $\wedge R^{6}$. The symmetry group of a pattern is

$$
\begin{aligned}
H & =G \cap\{1, \gamma\}\left\{\Omega(6) \wedge Z^{6}\right\}\{1, \gamma\}^{-1} \\
& =\{1, \gamma\}\left\{A(5) \wedge Z^{6}\right\}\{1, \gamma\}^{-1},
\end{aligned}
$$

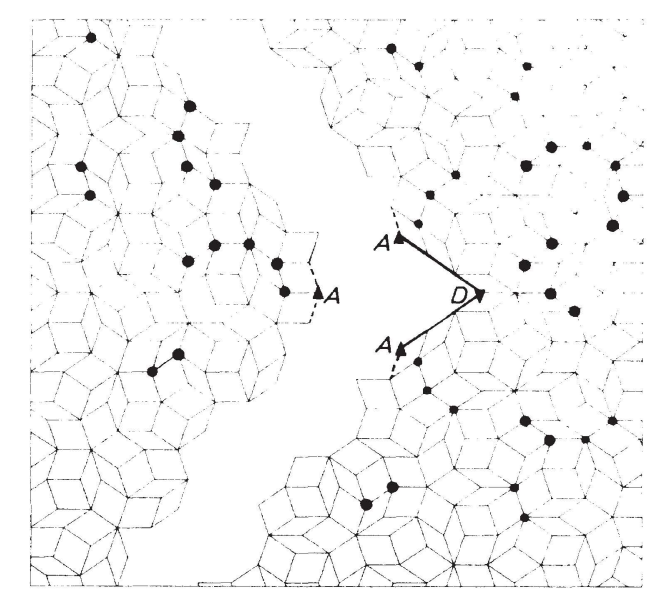

FIG. 3. Equivalence between a dislocation of Burgers vector $\mathrm{b}=(0,0,3,-3,0)$ and a $72^{\circ}$-disclination dipole. The two cores are marked $(A=$ antidisclination, the three points have to be identified; $D=$ disclination). 
where $\Omega(6)$ denotes the six-dimensional hyperoctahedral group and $A(5)$ the icosahedral group. ${ }^{16}$ The fundamental group of the order-parameter space $G / H$ is $\pi_{1}(G / H)=\{1, \gamma\}\left\{\bar{A}(5) \wedge Z^{6}\right\}\{1, \gamma\}^{-1} . \bar{A}(5)$ denotes the lift of the icosahedral group into SU(2). ${ }^{10}$ The fundamental group classifies dislocation and disclination lines. The Volterra process proceeds as in the two-dimensional case.

${ }^{1}$ D. Shechtman, I. Blech, D. Gratias, and J. W. Cahn, Phys. Rev. Lett. 53, 1951 (1984).

${ }^{2}$ D. Levine and P. J. Steinhardt, Phys. Rev. Lett. 53, 2477 (1984).

${ }^{3}$ K. Urban, N. Moser, and H. Kronmüller, Phys. Status Solidi (a) 91, 411 (1985).

${ }^{4}$ See, for example, D. R. Nelson and B. I. Halperin, Phys. Rev. B 19, 2457 (1979).

${ }^{5} \mathrm{R}$. Penrose, Math. Intelligencer 2, 32 (1979). Often, the name "Penrose pattern" is reserved for the special class of tilings obtained by Penrose. In this paper, we characterize with this name all possible tilings of pentagonal symmetry which arise from the projection of the five-dimensional hypercubic lattice. The examples in the figures are original Penrose patterns.

${ }^{6}$ N. G. de Bruijn, Proc. K. Ned. Akad. Wet. Ser. A 84, 39 (1981); 84, 51 (1981).

${ }^{7}$ See, for example, A. L. Mackay, Physica (Amsterdam) A 114, 609 (1982).

${ }^{8}$ M. Duneau and A. Katz, Phys. Rev. Lett. 54, 2688 (1985); A. Katz and M. Duneau, J. Phys. (Paris) 47, 181 (1986).

${ }^{9}$ P. Kramer and R. Neri, Acta Crystallogr. Sect. A 40, 580 (1984).

${ }^{10}$ For a review, see, for example, N. D. Mermin, Rev. Mod. Phys. 51, 591 (1979); H.-R. Trebin, Adv. Phys. 31, 195 (1982). These references also discuss the validity of the topological defect classification for crystals.

${ }^{11}$ Equivalent to our "active" viewpoint is the "passive" one (Ref. 8), where the five-dimensional lattice is fixed and the strip is shifted.

${ }^{12}$ D. Levine and P. J. Steinhardt, Phys. Rev. B 34, 596 (1986); J. E. S. Socolar and P. J. Steinhardt, Phys. Rev. B 34, 617 (1986).

${ }^{13}$ M. Klèman, Y. Gefen, and A. Pavlovitch, Europhys. Lett. 1, 61 (1986).

14 J. E. S. Socolar, T. Lubensky, and P. J. Steinhardt, Phys. Rev. B 34, 3345 (1986).

15J. P. Lu and J. L. Birman, Phys. Rev. Let. 57, 2701 (1986).

16P. Kramer, Z. Naturforsch. 40a, 775 (1985). 